\title{
HER2 expression and its clinicopathological features in resectable gastric cancer
}

\author{
Yoshiki Kataoka $\cdot$ Hiroshi Okabe $\cdot$ Akihiko Yoshizawa $\cdot$ \\ Sachiko Minamiguchi $\cdot$ Kenichi Yoshimura • \\ Hironori Haga · Yoshiharu Sakai
}

Received: 8 October 2011/Accepted: 16 February 2012/Published online: 14 March 2012

(c) The International Gastric Cancer Association and The Japanese Gastric Cancer Association 2012

\begin{abstract}
Background A recent randomized controlled trial (Trastuzumab for Gastric Cancer [ToGA] study) established standard scoring criteria of human epidermal growth factor receptor 2 (HER2) for gastric cancer and demonstrated the efficacy of trastuzumab for treating metastatic gastric cancer. The aim of the present study was to evaluate the frequency of HER2-positive cases by application of the standard criteria in patients with resectable gastric cancer and to examine the relationships between HER2 expression and prognosis, mucin phenotype, p53 status, and clinicopathological features.

Methods A total of 213 patients were included in this retrospective study. All tumor samples were examined for HER2 expression by immunohistochemistry (IHC), HER2 amplification by in situ hybridization, and mucin and $\mathrm{p} 53$ expression by staining for CD10, MUC2, MUC5AC, MUC6, and p53.
\end{abstract}

Y. Kataoka $\cdot$ H. Okabe $(\bowtie) \cdot$ Y. Sakai

Department of Surgery, Graduate School of Medicine,

Kyoto University, 54 Shogoin Kawahara-Cho, Sakyo-ku,

Kyoto 606-8507, Japan

e-mail: hokabe@kuhp.kyoto-u.ac.jp

A. Yoshizawa

Department of Laboratory Medicine,

Shinshu University Hospital, Matsumoto, Japan

A. Yoshizawa $\cdot$ S. Minamiguchi $\cdot$ H. Haga

Department of Diagnostic Pathology,

Kyoto University Hospital, Kyoto, Japan

K. Yoshimura

Translational Research Center,

Kyoto University Hospital, Kyoto, Japan
Results HER2-positive tumors were identified in 25 patients $(11.7 \%)$. HER2-positive cases were more frequently found in men, older patients, and in the intestinal histological type $(P=0.0048,0.0309$, and $<0.0001$, respectively). Although no association was found between HER2 overexpression and mucin phenotype, the expression of CD10 and p53 was significantly correlated with HER2 positivity $(P=0.0079$ and 0.013$)$. The overall survival of HER2-negative and -positive patients was not significantly different. However, in patients with stage III/ IV, overall survival was worse in HER2-positive patients $(P=0.0149)$. In a comparison between dual-color in situ hybridization (DISH) and fluorescence in situ hybridization (FISH), four IHC $2+/ 3+$ cases that were DISH-positive were judged as negative by FISH.

Conclusions Our study indicated that HER2 expression was less frequent in resectable gastric cancer than in metastatic gastric cancer. The impact of HER2 expression on survival was limited. DISH was superior to FISH for evaluating cases with limited HER2 expression.

Keywords Human epidermal growth factor receptor 2 . Gastric cancer - Immunohistochemistry .

In situ hybridization $\cdot$ Mucin phenotype

\section{Introduction}

The clinical benefit of trastuzumab was shown in an international phase III randomized controlled trial in patients with inoperable or metastatic human epidermal growth factor receptor 2 (HER2)-positive advanced gastric or gastroesophageal junction cancer [Trastuzumab for Gastric Cancer study (ToGA study)] [1]. Although many studies have previously evaluated HER2 status in gastric 
cancer, the patient cohorts and scoring criteria have varied, resulting in discrepancies in HER2 positivity that have ranged from 8.2 to $53.4 \%$ [2]. Frequent heterogeneity of HER2 status in gastric adenocarcinoma has also made the diagnosis of HER2 overexpression difficult and nonreproducible. To solve these problems, the ToGA study employed a new set of immunohistochemistry (IHC) scoring criteria, which was developed based on the study by Hofmann et al. [3] and which considers the biological features of gastric cancer. Using the new criteria, the ToGA study found HER2-positive tumors in $22.1 \%$ of metastatic gastric cancer cases.

The efficacy of trastuzumab for treating metastatic gastric cancer was clearly demonstrated in the ToGA study, suggesting that anti-HER2 therapy is also promising for resectable HER2-positive gastric cancer. However, the frequency of HER2-positive tumors determined by the new criteria in resectable gastric cancer has not been examined. To design a proper trial protocol of neoadjuvant or adjuvant therapy using trastuzumab for resectable HER2-positive gastric cancer, the frequency of HER2 positivity in resectable gastric cancer needs to be determined.

Some studies have reported that HER2 expression is associated with poorer prognosis in gastric cancer [4-12], although a direct correlation has not been proven [13-19]. Interpretation of these controversial results is difficult, because each study used a different definition of HER2 overexpression or amplification. Regarding the clinicopathological features of HER2-positive gastric cancer, HER2 expression and intestinal histological type have shown a high correlation. When focusing on the cellular origin or differentiation of gastric adenocarcinoma, the expressions of different types of mucins are used as epithelial differentiation markers: MUC5AC and MUC6 as gastric cell markers, and CD10 and MUC2 as intestinal markers [20]. The development of HER2-positive tumors could be linked to the particular type of differentiation. However, no study has investigated the relationship between HER2-positive gastric cancer and mucin phenotype. The overexpression of the mutated p53 gene is a major genetic event in gastric carcinogensis [21]. Although some studies have shown a correlation between p53 nuclear staining and HER2 expression, little is known about the relationship between p53 overexpression and HER2 positivity or mucin phenotype [17, 22].

The purpose of this study was to evaluate the frequency of HER2-positive gastric cancer, by applying the standard scoring criteria in patients with curatively resected gastric cancer. The relationships between HER2 expression and prognosis, mucin phenotype, $\mathrm{p} 53$ overexpression, and other clinicopathological features were also examined. Finally, we discuss the heterogeneity of HER2 overexpression in gastric cancer with a careful review of cases with discordance of HER2 overexpression and gene amplification, and we discuss two different hybridization methods, fluorescence in situ hybridization (FISH) and dual-color in situ hybridization (DISH).

\section{Patients and methods}

\section{Patients}

Among patients who underwent curative resection for primary gastric cancer at the Kyoto University Hospital between January 2001 and December 2007, 242 patients were diagnosed with pathological TNM stage IB-IV. Excluding 29 patients who received neoadjuvant chemotherapy, a total of 213 patients were included in this retrospective study. Sixty of these 213 patients received adjuvant chemotherapy. The study protocol was approved by the institutional review board. Clinicopathological parameters, including age, gender, tumor location, histological classification, pathological TNM stage, and lymphovascular invasion status were retrieved from medical charts or pathology reports. Histological classification was determined according to Lauren's classification, and the World Health Organization (WHO) classification. In the WHO classification, tubular adenocarcinoma with a poorly differentiated variant in more than half of the tumor is defined as mixed carcinoma, and if less than half, tubular adenocarcinoma.

\section{Evaluation of HER2 expression and amplification}

All tissues were fixed with $10 \%$ buffered formalin for 24-72 h, and then paraffin-embedded. Sections, 3- $\mu \mathrm{m}$ thick, were cut from a paraffin block of each specimen and submitted to DISH, hematoxylin and eosin staining, and IHC of HER2. Of the 213 cases, 32 with $\mathrm{IHC} 2+/ 3+$ or $\mathrm{DISH}+$, and 43 randomly selected from among the IHC $0 / 1+$ cases, were evaluated by FISH. IHC staining of HER2 with PATHWAY ${ }^{\circledR}$ HER2/neu (4B5) antibody (Ventana Medical Systems, Tucson, AZ, USA) was performed using an automated slide stainer (Bench-Mark XT; Ventana Medical Systems). As 4B5 stains invariably show extensive cytoplasmic background staining of the gastric foveolar layer and intestinal metaplasia, HER2 IHC was evaluated according to the stepwise process proposed by Rüschoff et al. [23]. For IHC scoring, the scoring scheme of the ToGA study was employed [1].

DISH was performed using the INFORM Dual ISH HER2 kit (Ventana Medical Systems). HER2 IHC and DISH were evaluated by an investigator (Y.K.) and a pathologist (S.M.). Positivity for HER2 was defined as either IHC3+ or IHC2+ with DISH+. FISH analysis was 
carried out using the PathVysion HER-2 DNA Probe Kit (Abbott, Des Plaines, Il, USA) after pretreatment with the Paraffin Pretreatment Kit (Abbott). FISH was evaluated by an investigator (Y.K.). Nuclei of invasive tumor cells were scored using a Biozero 8000 microscope (Keyence, Osaka, Japan) equipped with 4',6-diamidino-2-phenylindole (DAPI)/Green/Orange triple bandpass filters. In DISH and FISH, the HER2/chromosome 17 (Chr17) ratio was determined by counting the HER2 signals and Chr17 signals in 20 nuclei. Amplification of the $H E R 2$ gene was defined as a HER2/Chr17 ratio higher than 2.2. Negativity for HER2 amplification was defined as a HER2/Chr17 ratio of $<1.8$. When a ratio was between 1.8 and 2.2, signals in another 20 nuclei were counted, and the HER2/Chr17 ratio in a total of 40 nuclei was determined. When the ratio was $\geq 2.0$, amplification was defined as positive; otherwise it was defined as negative.

Mucin phenotype and p53 expression

Mucin and p53 IHC staining was performed by the tyramide signal amplification-avidin-biotin complex method [24]. We used monoclonal antibodies against MUC5AC (Novocastra, Newcastle-upon-Tyne, UK; diluted 1:100) as a marker for gastric foveolar cells, MUC6 (Novocastra; 1:100) as a marker for gastric mucous neck cells and pyloric glands, MUC2 (Novocastra; 1:100) as a marker for intestinal goblet cells, CD10 (Novocastra; 1:100) as a marker for the small intestinal brush border, and p53 (Novocastra, NCL-p53-Do7).

The expressions of CD10, MUC2, MUC5AC, and MUC6 were regarded as positive when more than $10 \%$ of the area was positively stained [20]. Overexpression of p53 was regarded as positive when more than $10 \%$ of tumor cells displayed nuclear immunostaining [25]. The phenotypes were classified into four categories according to combinations of the expressions of CD10 (brush border), MUC2 (goblet cells), MUC5AC (gastric foveolar epithelium), and MUC6 (mucous neck cells, pyloric glands). The intestinal (I) phenotype exhibited the expression of either CD10 or MUC2 but not of MUC5AC or MUC6. The gastrointestinal (GI) phenotype exhibited the expression of either CD10 or MUC2, in addition to the expression of either MUC5AC or MUC6. The gastric (G) phenotype exhibited the expression of either MUC5AC or MUC6 but not of CD10 or MUC2. The unclassified (U) phenotype exhibited no expression of CD10, MUC2, MUC5AC, or MUC6.

\section{Recurrence patterns}

Recurrence patterns were classified as locoregional, peritoneal, or hematogenous [26]. Locoregional recurrence was defined as any cancer recurrence at the resection margin or lymph nodes (LNs) (including regional nodes, as well as retroperitoneal, retropancreatic, para-aortic, and Virchow's nodes). Peritoneal recurrence was defined as any cancer recurrence within the abdominal cavity due to intraperitoneal distribution, including the rectal shelf. Hematogenous recurrence was defined as any metastatic lesion detected in liver, lung, ovary, adrenal gland, or bone.

Statistical analysis

All statistical analyses were conducted using the JMP 9.0.0 statistical software program (SAS Institute, Cary, NC, USA). The Pearson $\chi^{2}$ test and Wilcoxon test were performed to assess the correlation of clinicopathological parameters with HER2 positivity. All $P$ values were twosided, and $P<0.05$ was considered statistically significant. Survival curves were plotted using the Kaplan-Meier method, and the significance of differences between survival curves was evaluated using the log-rank test.

\section{Results}

HER2 positivity and clinical factors

The results for HER2 IHC and DISH in 213 patients are shown in Table 1 . When HER2 positivity was defined as IHC3 , or IHC2+ and DISH+, 25 out of the 213 patients $(11.7 \%)$ were diagnosed as HER2-positive. The association of HER2 positivity and clinicopathological features is

Table 1 Comparison of IHC and DISH test results for HER2 status

\begin{tabular}{llllll}
\hline & IHC $3+$ & IHC $2+$ & IHC $1+$ & IHC 0 & Total \\
\hline DISH+ & 18 & 7 & 0 & 1 & $26(12.2 \%)$ \\
DISH - & 0 & 6 & 18 & 163 & $187(87.8 \%)$ \\
Total & $18(8.5 \%)$ & $13(6.1 \%)$ & $18(8.5 \%)$ & $164(77.0 \%)$ & 213 \\
\hline
\end{tabular}

IHC scores are based on the ToGA HER2 scoring scheme

IHC immunohistochemistry, DISH dual-color in situ hybridization, HER2 human epidermal growth factor receptor 2, ToGA Trastuzumab for Gastric Cancer 
Table 2 Comparison of clinicopathological factors between HER2negative and HER2-positive gastric cancer

\begin{tabular}{|c|c|c|c|}
\hline \multirow[t]{2}{*}{ Variable } & \multicolumn{2}{|l|}{ HER2 status } & \multirow{2}{*}{$P$ value } \\
\hline & $\begin{array}{l}\text { Positive }(\%) \\
(n=25)\end{array}$ & $\begin{array}{l}\text { Negative }(\%) \\
(n=188)\end{array}$ & \\
\hline Age (years) ${ }^{\mathrm{a}}$ & $71.2 \pm 9.5$ & $66.2 \pm 11.2$ & 0.0309 \\
\hline Gender & & & 0.0048 \\
\hline Male & $23(16.1)$ & $120(83.9)$ & \\
\hline Female & $2(2.86)$ & $68(97.1)$ & \\
\hline Tumor location & & & 0.8387 \\
\hline Upper & $8(13.8)$ & $50(86.2)$ & \\
\hline Middle & $11(12.2)$ & $79(87.9)$ & \\
\hline Lower & $5(8.6)$ & $53(91.4)$ & \\
\hline Other & $1(14.3)$ & $6(85.7)$ & \\
\hline WHO classification & & & $<0.0001$ \\
\hline Tubular & $20(21.7)$ & $72(78.3)$ & \\
\hline Papillary & $1(50.0)$ & $1(50.0)$ & \\
\hline Mucinous & $0(0)$ & $5(100)$ & \\
\hline Poorly cohesive & $0(0)$ & $88(100)$ & \\
\hline Mixed & $4(15.4)$ & $22(84.6)$ & \\
\hline Lauren's classification & & & $<0.0001$ \\
\hline Intestinal & $21(22.3)$ & $73(77.7)$ & \\
\hline Mixed & $4(15.4)$ & $22(84.6)$ & \\
\hline Diffuse & $0(0)$ & $93(100)$ & \\
\hline Depth of tumor (T) & & & 0.8666 \\
\hline $1 \mathrm{a}$ & $1(25.0)$ & $3(75.0)$ & \\
\hline $1 b$ & $3(13.6)$ & $19(86.4)$ & \\
\hline 2 & $9(13.2)$ & $59(86.8)$ & \\
\hline 3 & $8(11.4)$ & $62(88.6)$ & \\
\hline $4 a$ & $4(9.1)$ & $40(90.9)$ & \\
\hline $4 \mathrm{~b}$ & $0(0)$ & $5(100)$ & \\
\hline Nodal stage $(\mathrm{N})$ & & & 0.9052 \\
\hline 0 & $6(8.8)$ & $62(91.2)$ & \\
\hline 1 & 7 (14.6) & $41(85.4)$ & \\
\hline 2 & $7(13.0)$ & $47(87.0)$ & \\
\hline $3 a$ & $3(11.1)$ & $24(88.9)$ & \\
\hline $3 b$ & $2(12.5)$ & $14(87.5)$ & \\
\hline TNM stage & & & 0.7816 \\
\hline IB & $5(10.0)$ & $45(90.0)$ & \\
\hline IIA & $8(16.7)$ & $40(83.3)$ & \\
\hline IIB & $3(10.3)$ & $26(89.7)$ & \\
\hline IIIA & $3(10.3)$ & $36(89.7)$ & \\
\hline IIIB & $5(15.2)$ & $28(84.9)$ & \\
\hline IIIC & $1(5.6)$ & $17(94.4)$ & \\
\hline IV & $0(0)$ & $6(100)$ & \\
\hline Lymphatic invasion & & & 0.2754 \\
\hline Absent & $11(15.1)$ & $62(84.9)$ & \\
\hline Present & $14(10.0)$ & $126(90.0)$ & \\
\hline Venous invasion & & & 0.2150 \\
\hline Absent & $10(9.1)$ & $100(90.9)$ & \\
\hline Present & 15 (14.6) & $88(85.4)$ & \\
\hline
\end{tabular}

Table 2 continued

\begin{tabular}{llll}
\hline Variable & \begin{tabular}{l} 
HER2 status \\
\cline { 2 - 3 }
\end{tabular} & $\begin{array}{l}\text { Positive }(\%) \\
(n=25)\end{array}$ & $\begin{array}{l}\text { Negative }(\%) \\
(n=188)\end{array}$ \\
\hline $\begin{array}{l}\text { Recurrence } \\
\text { Absent }\end{array}$ & $17(11.6)$ & $130(88.4)$ & 0.9071 \\
Present & $8(12.1)$ & $58(87.9)$ & \\
\hline
\end{tabular}

$P$ values were calculated using $\chi^{2}$ tests for categorical variables and Wilcoxon tests for continuous variables. Percentages show the ratios of HER2-positive or -negative patients for each item

HER2 human epidermal growth factor receptor 2, WHO World Health Organization

${ }^{a}$ Age was reported as the mean $\pm \mathrm{SD}$

summarized in Table 2. HER2-positive cases were more frequently found in men $(P=0.0048)$ and older patients $(P=0.0309)$. HER2-positive tumors were found twice as often in upper-third compared to lower-third lesions (13.8 vs. $8.6 \%$ ). Notably, HER2-positive tumors were more frequently found among tumors invading the esophagus than in other tumors (four out of 12 tumors [33.3\%] vs. $10.4 \%, P=0.0167)$. In terms of histology, HER2-positive gastric cancer showed predominantly papillary, tubular, and mixed adenocarcinoma according to the WHO classification $(P<0.0001)$. According to Lauren's classification, HER2 overexpression was more often detected in the intestinal histological type $(22.3 \%)$, than in the mixed $(15.4 \%)$ or diffuse (0 \%) type. All four mixed-type HER2positive cases displayed HER2 IHC positivity in the intestinal component, supporting the strong correlation between HER2 expression and intestinal histological type. HER2-positive tumors tended to present with venous invasion, although this tendency was not statistically significant $(P=0.2150)$. No correlation was found between HER2 positivity and T or $\mathrm{N}$ factors, or the TNM stage.

HER2 and mucin phenotypic classification

The results of the analysis of the expression of the four mucin markers and phenotypic classification based on mucin expression are shown in Table 3. Of the four markers, CD10 expression was significantly correlated with HER2 positivity $(P=0.0079)$. A representative example of a case positive for HER2 and CD10 is shown in Fig. 1. There was no correlation between the other three mucin markers and HER2 overexpression. When the mucin phenotype was classified into four subtypes, the HER2positive ratios in the I, G, and GI phenotypes were 12.2, 13.9 , and $13.2 \%$, whereas only one case of the U phenotype exhibited HER2 positivity (3.2\%); however, the difference between the phenotypes was not significant. 
Table 3 Comparison of HER2 status and mucin expression or phenotypic classification

\begin{tabular}{|c|c|c|c|}
\hline & \multicolumn{2}{|l|}{ HER2 status } & \multirow[t]{2}{*}{$P$ value } \\
\hline & $\begin{array}{l}\text { Positive }(\%) \\
(n=25)\end{array}$ & $\begin{array}{l}\text { Negative }(\%) \\
(n=188)\end{array}$ & \\
\hline \multicolumn{4}{|l|}{ Mucin expression } \\
\hline CD10 & & & 0.0079 \\
\hline Negative & $14(8.5)$ & $150(91.5)$ & \\
\hline Positive & $11(22.5)$ & $38(77.6)$ & \\
\hline MUC2 & & & 0.8870 \\
\hline Negative & $15(12.0)$ & $110(88.0)$ & \\
\hline Positive & $10(11.4)$ & $78(88.6)$ & \\
\hline MUC5AC & & & 0.9711 \\
\hline Negative & $11(11.8)$ & $82(88.2)$ & \\
\hline Positive & $14(11.7)$ & $106(88.3)$ & \\
\hline MUC6 & & & 0.5207 \\
\hline Negative & $15(10.7)$ & $125(89.3)$ & \\
\hline Positive & $10(13.7)$ & $63(86.3)$ & \\
\hline Phenotypic classification & & & 0.4564 \\
\hline Intestinal (I) & $6(12.2)$ & $43(87.8)$ & \\
\hline Gastric (G) & $9(13.9)$ & $56(86.2)$ & \\
\hline Gastrointestinal (GI) & $9(13.2)$ & $59(86.8)$ & \\
\hline Unclassified (U) & $1(3.2)$ & $30(96.8)$ & \\
\hline
\end{tabular}

$P$ values were calculated using $\chi^{2}$ tests

HER2 human epidermal growth factor receptor 2

Neither the expression of any type of mucin nor the mucin phenotype was associated with the patients' overall survival (OS) or recurrence-free survival (RFS) (data not shown).

\section{HER2 and p53 overexpression}

Overexpression of p53 was detected in $75(35.2 \%)$ of the cases, being significantly correlated with HER 2 positivity $(P=0.013) ; \mathrm{p} 53$ overexpression was found in 16 of 25 HER-2-positive tumors (64.0\%), and in 59 of 188 HER-2 negative tumors $(31.4 \%)$. p53 overexpression was also detected more often in the intestinal than in the diffuse/ mixed type (54.3 vs. $20.2 \%: P<0.0001)$. Regarding the relationship between p53 and markers of mucin phenotype, p53 overexpression tended to be found more frequently in CD10-positive than in CD10-negative tumors (46.9 vs. $31.7 \%: P=0.0501)$.

HER2 and survival

Kaplan-Meier curves for OS and RFS constructed according to differences in HER2 status are shown in Fig. 2a, b. Although the survival curves of HER2-positive patients were slightly worse than those of HER2-negative patients, the difference was not significant (OS: $P=$ 0.2203 , RFS: $P=0.1996)$. When patients were stratified into TNM stages IB/II and III/IV, no correlation was found between HER 2 status and OS in the stage IB/II group $(P=0.6060)$. However, in patients with stage III/IV, the OS of patients with a HER2-positive tumor was significantly worse than that of the patients with a HER2-negative tumor $(P=0.0149)$ (Fig. 2c, d).

In the HER2-positive patients with stage III/IV, the most common pattern of recurrence was hematogenous ( 3 cases, $60 \%$ ), followed by locoregional ( 1 case, $20 \%$ ) and peritoneal (1 case, $20 \%)$ recurrence. In the HER2-negative patients with stage III/IV, the most common pattern was locoregional ( 15 cases, $35.7 \%$ ), followed by peritoneal (14 cases, $33.3 \%)$ and hematogenous (13 cases, $31.0 \%)$ recurrence. Patterns of recurrence were not significantly different between the two groups $(P=0.4316)$.

Diagnosis of HER2 positivity and HER2 heterogeneity

A summary of the assessment of IHC scoring and HER2 amplification by in situ hybridization in 75 cases is shown in Table 4. When IHC2+/3+ was defined as IHC-positive, and $\mathrm{IHC} 1+/ 0$ was defined as IHC-negative, the overall concordance rate between IHC and DISH was $96.7 \%$. Among 13 tumors with equivocal IHC results (IHC2+), DISH was positive in seven tumors $(53.8 \%)$. When these IHC2 + cases were excluded, DISH was positive in all IHC3 + patients, and only one of the IHC-negative patients showed HER2 amplification. Of the 75 samples, four could not be assessed by FISH because of technical difficulties ( 2 cases with IHC0/DISH-, 1 case with IHC2+/DISH+, and 1 case with $\mathrm{IHC} 0$ and DISH+). On comparing the results of DISH and FISH analyses, four cases (5.6\%) of inconsistent results were identified. Three IHC3+ cases and one IHC2+ case positive for DISH were judged as negative by FISH.

Table 5 shows the ratio of HER2-stained cells with IHC2+ and $3+$ in the HER2-positive cases. Of 25 tumors, only two $(8 \%)$ stained $100 \%$ positive for HER2. Comparing the area of HER2 expression and HER2 amplification, in the majority of HER2-positive cases, HER2 amplification was observed in the area positively stained by IHC for HER2. However, HER2 amplification was occasionally identified in the HER2-negative area.

\section{Discussion}

This study included 213 patients with curative resection of primary gastric cancer, and HER2 expression was assessed using the scoring scheme employed in the ToGA study [1]. We defined HER 2 positivity as $\mathrm{IHC} 3+$, or $\mathrm{IHC} 2+$ and $\mathrm{DISH}+$, because the clinical benefit of trastuzumab in this 

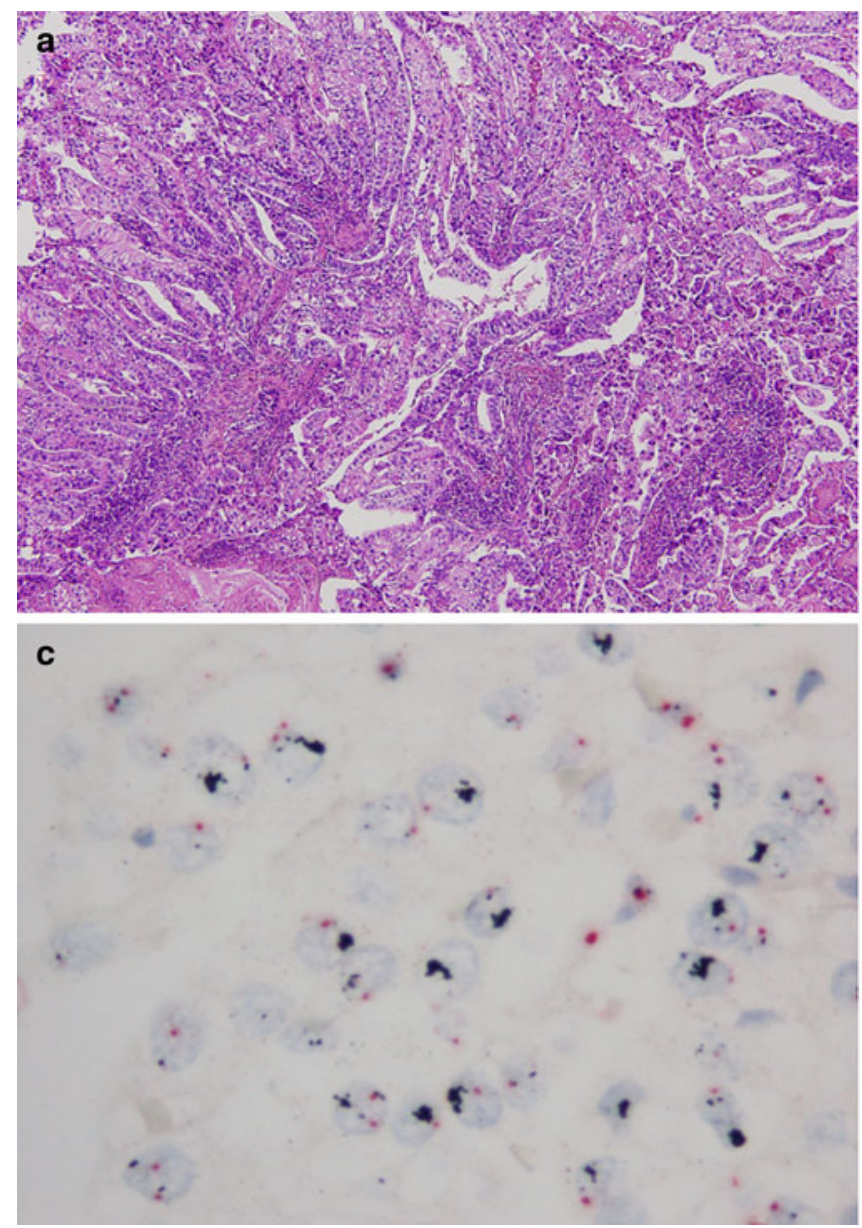

Fig. 1 A representative case of human epidermal growth factor receptor 2 (HER2)-positive gastric cancer with CD10 expression. a Specimen showing intestinal-type adenocarcinoma $(\mathrm{H} \& \mathrm{E}, \times 100)$. b Specimen showing strong basolateral membranous HER2 immunoreactivity, which is scored as $3+($ HER2-IHC, $\times 100)$. c Specimen showing HER2 gene amplification with a HER2/chromosome 17

subgroup was evident. When the same definition was applied to the patients in the ToGA study, the HER2positive ratio was estimated to be $17.1 \%$, whereas the present study identified 25 HER2-positive cases, accounting for $11.7 \%$ of all included cases. This difference in the HER2-positive ratio between the two studies may be attributed to the different backgrounds of the patients. That is, the ToGA study population consisted only of patients with metastatic or recurrent gastric cancer, while the present study population consisted of patients with curatively resected gastric cancer. A recent study has reported a HER2-positive ratio of $8.1 \%$ for curatively resected gastric cancer [16], similar to our finding. Taken together, these results suggest that HER2-positive gastric cancer might be less frequent in resectable gastric cancer than in metastatic cases.

Four large studies have assessed HER2 expression and survival in gastric cancer patients, two of which have
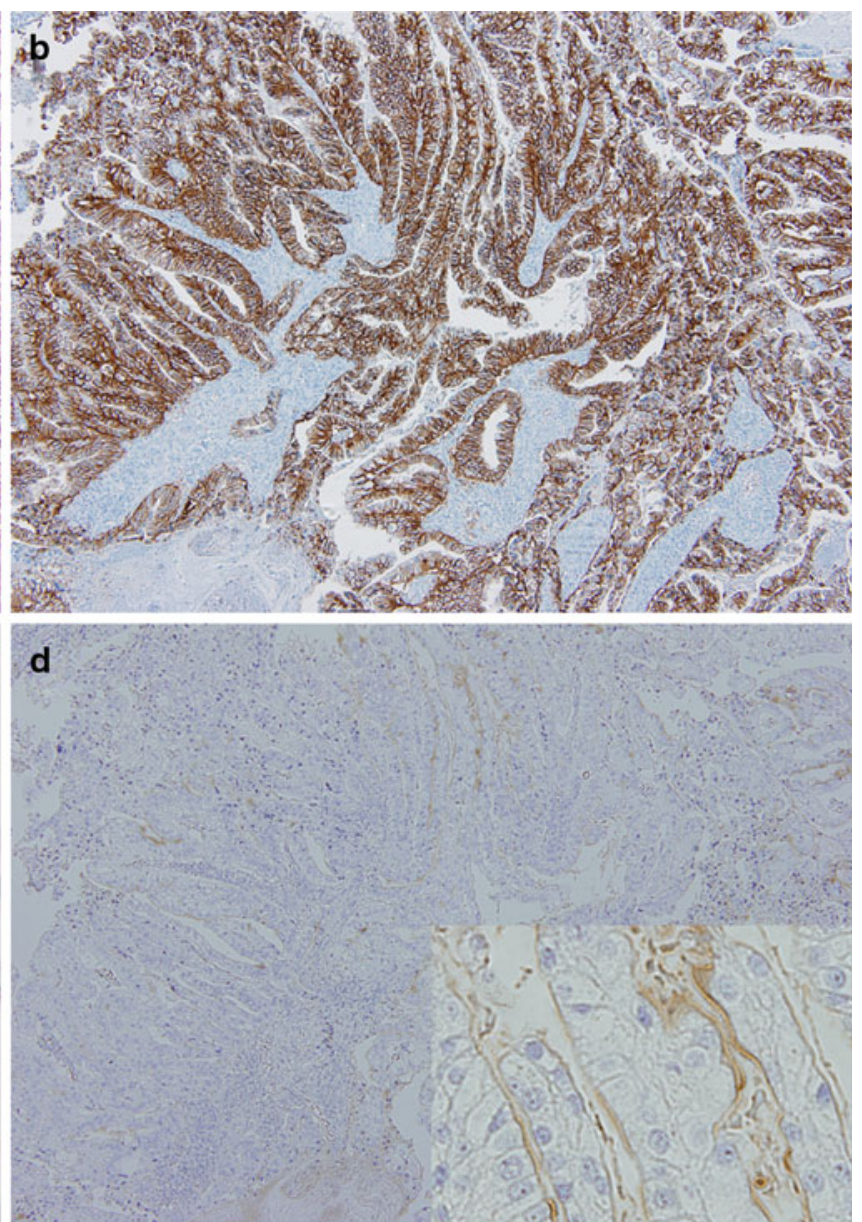

[Chr17] ratio of 5.62 (HER2-dual-color in situ hybridization [DISH], $\times 1000$ ). Black and red signals represent HER2 and Chr17, respectively. d Specimen showing CD10 immunoreactivity along the luminal surface of the carcinoma glands $(\mathrm{CD} 10, \times 100$; inset original magnification $\times 400$ )

shown no association between these parameters [14, 16]. The other two studies analyzed only intestinal-type gastric cancer and demonstrated poorer prognosis in HER2-positive cases $[13,27]$. In our study, patient survival was not significantly different between HER2-positive and -negative cases, even when the analysis included only intestinaltype gastric cancer. Although the ratio of HER2-positive cases was nearly half of the value that we had hypothesized, our study still maintained enough statistical power to detect the general impact of HER2 expression on survival. Thus, in contrast to breast cancer, in which HER2 overexpression is an established strong prognostic factor, HER2 status may not be a distinct prognostic factor in resectable gastric cancer. However, it is possible that HER2 is a prognostic factor only for advanced disease, because in the TNM stage III/IV subgroup, the survival of HER2-positive patients was significantly shorter than that of the HER2negative patients. By applying the standardized scoring 
Fig. 2 Kaplan-Meier curves of HER2-positive and -negative patients for overall survival (a), recurrence-free survival (b), overall survival in TNM stage IB/II (c), and overall survival in TNM stage III/IV (d)
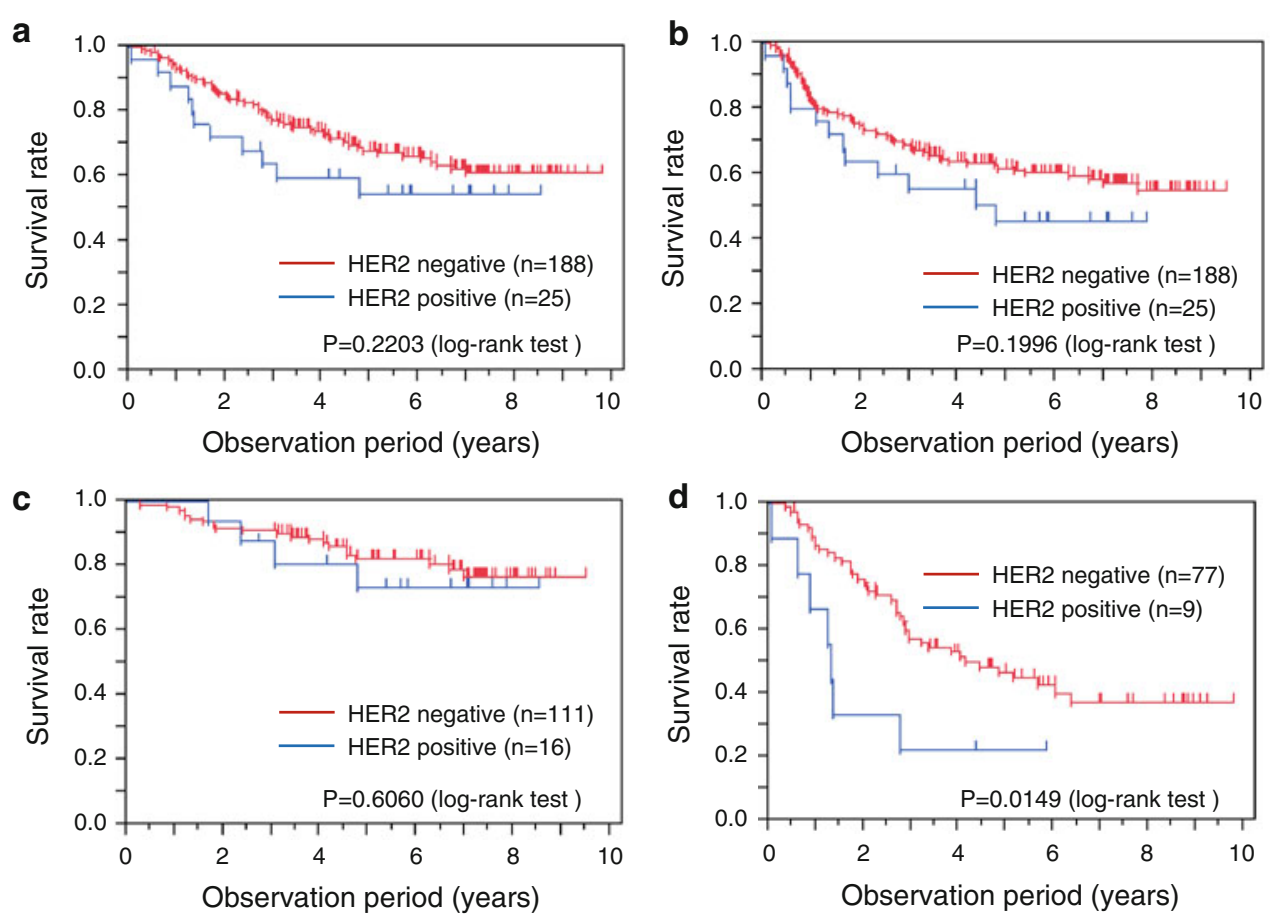

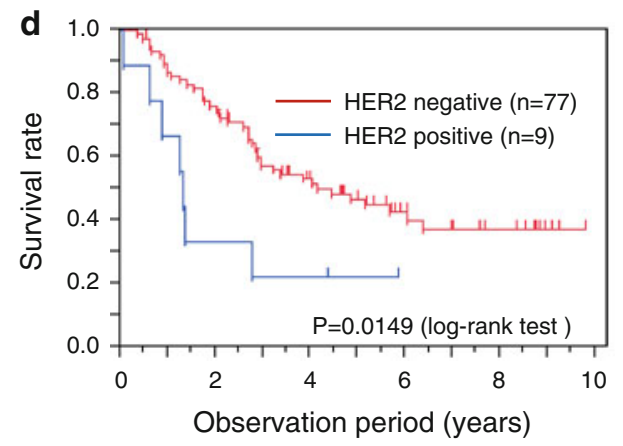

Table 4 Concordance between DISH and FISH results

\begin{tabular}{|c|c|c|c|c|c|c|}
\hline \multirow{2}{*}{$\begin{array}{l}\text { IHC } \\
\text { score }\end{array}$} & \multicolumn{2}{|l|}{ DISH } & \multicolumn{2}{|l|}{ FISH } & \multirow{2}{*}{$\begin{array}{l}\text { Not } \\
\text { available }\end{array}$} & \multirow[t]{2}{*}{ Tota } \\
\hline & Positive & Negative & Positive & Negative & & \\
\hline $3+$ & 18 & 0 & 15 & 3 & 0 & 18 \\
\hline $2+$ & 7 & 6 & 5 & 7 & 1 & 13 \\
\hline $1+$ & 0 & 6 & 0 & 6 & 0 & 6 \\
\hline 0 & 1 & 37 & 0 & 35 & 3 & 38 \\
\hline Total & 26 & 49 & 20 & 51 & 4 & $75^{\mathrm{a}}$ \\
\hline
\end{tabular}

DISH dual-color in situ hybridization, FISH fluorescence in situ hybridization, $I H C$ immunohistochemistry

${ }^{\text {a }}$ Four specimens were not available for FISH

Table 5 Ratio of HER2-stained cells in HER2-positive cases

\begin{tabular}{ll}
\hline Percentage $^{\mathrm{a}}$ & $n(\%)$ \\
\hline$\geq 90$ & $7(28.0)$ \\
50 to $<90$ & $9(36.0)$ \\
30 to $<50$ & $2(8.0)$ \\
10 to $<30$ & $7(28.0)$
\end{tabular}

HER2 human epidermal growth factor receptor 2, IHC immunohistochemistry

${ }^{\text {a }}$ Percentage of HER2-stained cells with IHC2+ and 3+

criteria for HER2 assessment in gastric cancer, it is hoped that further studies will provide distinct outcomes around this issue.

Of the 25 HER2-positive tumors in the present study, 21 were intestinal type and four were mixed type according to
Lauren's classification. These data are consistent with previous reports that the intestinal type showed a higher rate of HER2 positivity than the diffuse/mixed type [13, 14, 16, 27-31]. A strong correlation between HER2 positivity and intestinal histological type is also supported by our finding that even in the four HER2-positive, mixed-type cases, IHC staining of HER2 was positive only in the intestinal component. In the ToGA study, the HER2-positive ratio was higher in tumors at the gastroesophageal junction than in gastric cancer (33.2 vs. $20.9 \%$ ). Similarly, in our study, the HER2-positive ratio was almost three times as high in the tumors invading the esophagus than in the other lesions ( 33.3 vs. $10.4 \%$ ).

No correlation was found between HER2 positivity and $\mathrm{T}$ - or $\mathrm{N}$-factors, or TNM stage, in the present study. A HER2-positive tumor was even found in one of four T1a (tumor invades as far as the lamina propria or muscularis mucosae) cases. Previous studies including all pathological stages have also reported no correlation between pathological stage and HER2 overexpression [13, 14, 27, 31]. Taken together, these findings suggest that HER2 overexpression occurs in the early phase of gastric carcinogenesis. However, because there were small numbers of patients in the early stage in these reports, further studies are needed to determine the association between HER2 expression and the development of gastric cancer.

The present study is the first to have examined the association between the mucin phenotype and HER2 status of gastric cancer. Although we did not find a significant correlation between HER 2 status and the mucin phenotype, 


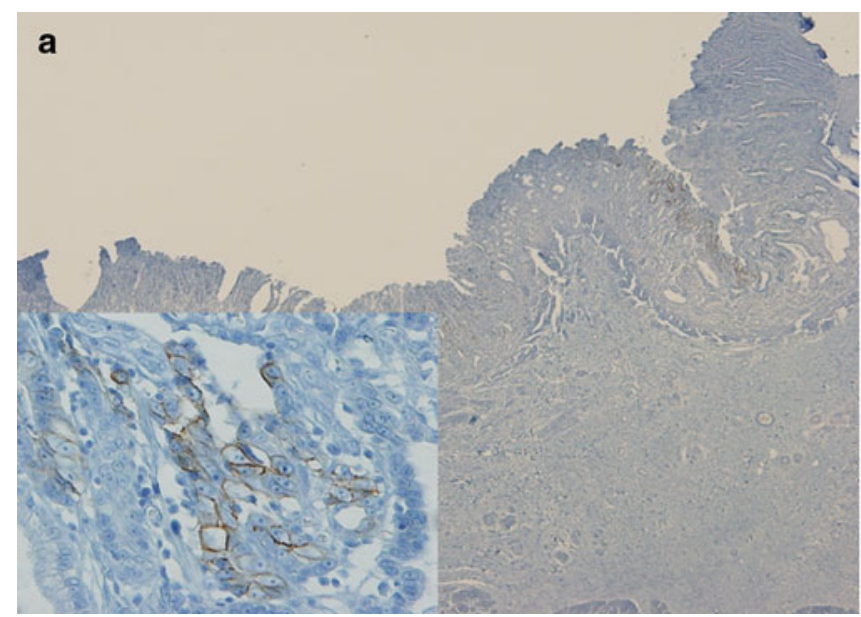

Fig. 3 HER2-negative case with immunohistochemistry (IHC) 2+stained cells in less than $5 \%$ of tumor cells and HER2 amplification by DISH in the IHC2+-stained area. a Specimen showing $\mathrm{IHC} 2+$ in

HER 2 expression was rarely detected in tumors without the expression of any type of mucin (unclassified or null type). This observation is consistent with previous reports that the HER2 positive ratio was lower in diffuse or undifferentiated-type gastric cancer. Of four mucin markers that we examined, the expression of CD10 (the marker for cells with small intestinal brush border differentiation) was significantly correlated with HER2 positivity. Because CD10 was strongly correlated with the intestinal histological type in our study $(P=0.0002)$, it seems that the correlation between $\mathrm{CD} 10$ and HER2 expression may reflect a linkage between the intestinal differentiation of cancer cells and HER2 expression. A correlation between intestinal phenotype and either a better or a poorer prognosis has been reported in some studies [20,32]. However, no significant difference in patient survival was observed according to the four different mucin phenotypes in the present study.

Accumulation of p53 protein in the nuclei of carcinoma cells is known to correlate well with the presence of mutations in the TP53 gene [33, 34]. Our study demonstrated a strong correlation between p53 overexpression and HER2 positivity, suggesting a possible role of TP53 abnormality in the development of HER2-positive gastric cancer. These findings are consistent with previous studies, which have reported a correlation between p53 nuclear staining and HER2 positivity [17, 22]. Intriguingly, some studies also reported a linkage between alterations of p53 and the intestinal histological type. Consistently, our study confirmed that p53 overexpression was more often found in the intestinal type of gastric cancer [17, 25]. These results suggest that the intestinal differentiation of cancer cells, as well as HER2 and CD10 expression, may also be linked to the expression of $\mathrm{p} 53$. The significance of the expression of

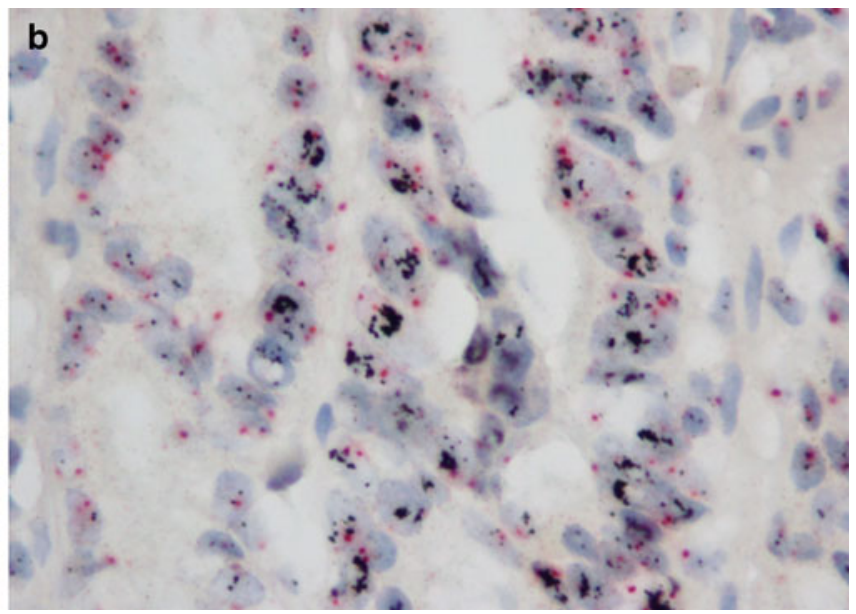

less than $5 \%$ of tumor cells (HER2-IHC, $\times 40$; inset original magnification $\times 400$ ). b Specimen showing high amplification in the IHC2+-stained area (HER2-DISH, $\times 1000)$

these molecules in regard to tumor biology and prognosis needs to be determined by further studies.

The concordance rate between IHC and DISH in the present study was as high as $96.7 \%$, which is similar to that in previously published studies $[3,13,14,16,27-31$, 35]. Especially in IHC3+ cases, amplification of the HER2 gene was confirmed by DISH in all cases. While HER2 is usually homogenously expressed in HER2-positive breast cancer, HER2 expression in gastric cancer is often known to be heterogeneous [36]. The majority of HER2-positive tumors in our study also exhibited heterogeneous expression of HER2, and the ratio of HER2-positive cells in HER2-positive cases varied (Table 5). In cases with smaller areas of HER2 expression, accurate diagnosis of HER2 using biopsy samples would be difficult, as a biopsy sample taken from a negative area would return a falsenegative result. Therefore, to improve the reliability of diagnosis in biopsy specimens, taking several samples from different parts of the tumor is recommended; however, the appropriate quantity or location for biopsy remains to be determined.

In IHC3 + cases, amplification of the HER2 gene was always confirmed by DISH, when the IHC-stained area was evaluated. Even in a case with HER2 expression in less than $5 \%$ of tumor cells, HER2 amplification was confirmed in the small IHC-stained area (Fig. 3). Because HER2 amplification is exclusively detected in the IHCstained area, to properly examine amplification, it is crucial to examine HER2 expression first. In IHC2+ cases, however, even when the HER2 expression area was properly assessed, amplification was detected in only half of the patients. Thus, to determine the HER2 status of IHC2+ cases, HER 2 amplification should also be evaluated. In our comparison of DISH and FISH, FISH failed to detect 
HER2 amplification in four IHC $3+/ 2+$ cases, which were correctly diagnosed by DISH. Two of these cases expressed HER 2 in less than $20 \%$ of tumor cells. In cases with a limited HER2 expression area, it may be easier to use DISH for examination of the proper area, because comparison with IHC by conventional microscopy is possible.

In conclusion, our study indicated that HER2 expression in resectable gastric cancer was less frequent than the expression in metastatic or recurrent gastric cancer. The impact of HER2 expression on patient survival was shown to be limited, especially in the earlier stages of the disease. When the heterogeneity of HER2 expression is taken into consideration, it seems that assessing whole tissue sections, or at least multiple biopsy samples, is necessary to make a proper diagnosis of HER2 status, and DISH proved to be superior to FISH for evaluating cases with limited HER2 expression. Further research is still need to clarify the relevance of HER2 heterogeneity for the clinical response to HER2 target therapy.

Acknowledgments We appreciate the technical support for FISH from Seiji Hashimoto. This study was partly supported by a grant from Chugai Pharmaceuticals.

\section{References}

1. Bang YJ, Van Cutsem E, Feyereislova A, Chung HC, Shen L, Sawaki A, et al. Trastuzumab in combination with chemotherapy versus chemotherapy alone for treatment of HER2-positive advanced gastric or gastro-oesophageal junction cancer (ToGA): a phase 3, open-label, randomised controlled trial. Lancet. 2010;376:687-97.

2. Jørgensen JT. Targeted HER2 treatment in advanced gastric cancer. Oncology. 2010;78:26-33.

3. Hofmann M, Stoss O, Shi D, Büttner R, van de Vijver M, Kim W, et al. Assessment of a HER2 scoring system for gastric cancer: results from a validation study. Histopathology. 2008;52:797-805.

4. Park DI, Yun JW, Park JH, Oh SJ, Kim HJ, Cho YK, et al. HER$2 /$ neu amplification is an independent prognostic factor in gastric cancer. Dig Dis Sci. 2006;51:1371-9.

5. Zhang XL, Yang YS, Xu DP, Qu JH, Guo MZ, Gong Y, et al. Comparative study on overexpression of HER2/neu and HER3 in gastric cancer. World J Surg. 2009;33:2112-8.

6. Uchino S, Tsuda H, Maruyama K, Kinoshita T, Sasako M, Saito $\mathrm{T}$, et al. Overexpression of c-erbB-2 protein in gastric cancer. Its correlation with long-term survival of patients. Cancer. 1993;72: 3179-84.

7. Nakajima M, Sawada H, Yamada Y, Watanabe A, Tatsumi M, Yamashita $\mathrm{J}$, et al. The prognostic significance of amplification and overexpression of c-met and c-erb B-2 in human gastric carcinomas. Cancer. 1999;85:1894-902.

8. Allgayer H, Babic R, Gruetzner KU, Tarabichi A, Schildberg FW, Heiss MM. c-erbB-2 is of independent prognostic relevance in gastric cancer and is associated with the expression of tumorassociated protease systems. J Clin Oncol. 2000;18:2201-9.

9. García I, Vizoso F, Martín A, Sanz L, Abdel-Lah O, Raigoso P, et al. Clinical significance of the epidermal growth factor receptor and HER2 receptor in resectable gastric cancer. Ann Surg Oncol. 2003;10:234-41.
10. Tanner M, Hollmén M, Junttila TT, Kapanen AI, Tommola S, Soini Y, et al. Amplification of HER-2 in gastric carcinoma: association with Topoisomerase IIalpha gene amplification, intestinal type, poor prognosis and sensitivity to trastuzumab. Ann Oncol. 2005;16:273-8.

11. Yonemura Y, Ninomiya I, Yamaguchi A, Fushida S, Kimura H, Ohoyama S, et al. Evaluation of immunoreactivity for erbB-2 protein as a marker of poor short term prognosis in gastric cancer. Cancer Res. 1991;51:1034-8.

12. Pinto-de-Sousa J, David L, Almeida R, Leitão D, Preto JR, Seixas $\mathrm{M}$, et al. c-erb B-2 expression is associated with tumor location and venous invasion and influences survival of patients with gastric carcinoma. Int J Surg Pathol. 2002;10:247-56.

13. Kim MA, Jung EJ, Lee HS, Lee HE, Jeon YK, Yang HK, et al. Evaluation of HER-2 gene status in gastric carcinoma using immunohistochemistry, fluorescence in situ hybridization, and real-time quantitative polymerase chain reaction. Hum Pathol. 2007;38:1386-93.

14. Marx AH, Tharun L, Muth J, Dancau AM, Simon R, Yekebas E, et al. HER-2 amplification is highly homogenous in gastric cancer. Hum Pathol. 2009;40:769-77.

15. Grabsch H, Sivakumar S, Gray S, Gabbert HE, Müller W. HER2 expression in gastric cancer: rare, heterogeneous and of no prognostic value-conclusions from 924 cases of two independent series. Cell Oncol. 2010;32:57-65.

16. Kunz PL, Mojtahed A, Fisher GA, Ford JM, Chang DT, Balise $\mathrm{RR}$, et al. HER2 Expression in gastric and gastroesophageal junction adenocarcinoma in a US population: clinicopathologic analysis with proposed approach to HER2 assessment. Appl Immunohistochem Mol Morphol. 2011;40(36):9212-20.

17. Lee KE, Lee HJ, Kim YH, Yu HJ, Yang HK, Kim WH, et al. Prognostic significance of p53, nm23, PCNA and c-erbB-2 in gastric cancer. Jpn J Clin Oncol. 2003;33:173-9.

18. Yu GZ, Chen Y, Wang JJ. Overexpression of Grb2/HER2 signaling in Chinese gastric cancer: their relationship with clinicopathological parameters and prognostic significance. J Cancer Res Clin Oncol. 2009;135:1331-9.

19. Barros-Silva JD, Leitão D, Afonso L, Vieira J, Dinis-Ribeiro M, Fragoso M, et al. Association of ERBB2 gene status with histopathological parameters and disease-specific survival in gastric carcinoma patients. Br J Cancer. 2009;100:487-93.

20. Wakatsuki K, Yamada Y, Narikiyo M, Ueno M, Takayama T, Tamaki H, et al. Clinicopathological and prognostic significance of mucin phenotype in gastric cancer. J Surg Oncol. 2008;98: 124-9.

21. Yokozaki H, Kuniyasu H, Kitadai Y, Nishimura K, Todo H, Ayhan A, et al. p53 point mutations in primary human gastric carcinomas. J Cancer Res Clin Oncol. 1992;119:67-70.

22. Al-Moundhri MS, Nirmala V, Al-Hadabi I, Al-Mawaly K, Burney I, Al-Nabhani M, et al. The prognostic significance of p53, p27 kip1, p21 waf1, HER-2/neu, and Ki67 proteins expression in gastric cancer: a clinicopathological and immunohistochemical study of 121 Arab patients. J Surg Oncol. 2005;91:243-52.

23. Rüschoff J, Dietel M, Baretton G, Arbogast S, Walch A, Monges $\mathrm{G}$, et al. HER2 diagnostics in gastric cancer-guideline validation and development of standardized immunohistochemical testing. Virchows Arch. 2010;457:299-307.

24. Toda Y, Kono K, Abiru H, Kokuryo K, Endo M, Yaegashi H, et al. Application of tyramide signal amplification system to immunohistochemistry: a potent method to localize antigens that are not detectable by ordinary method. Pathol Int. 1999;49: 479-83.

25. Ismail HM, Moneer M, El-Baradie M, Khorshid O, Touny A. Clinicopathologic and prognostic significance of overexpression of her-2/neu and p53 oncoproteins in gastric carcinoma using tissue microarray. J Egypt Natl Cancer Inst. 2007;19:147-57. 
26. Eom BW, Yoon H, Ryu KW, Lee JH, Cho SJ, Lee JY, et al. Predictors of timing and patterns of recurrence after curative resection for gastric cancer. Dig Surg. 2010;27:481-6.

27. Yan B, Yau EX, Bte Omar SS, Ong CW, Pang B, Yeoh KG, et al. A study of HER2 gene amplification and protein expression in gastric cancer. J Clin Pathol. 2010;63:839-42.

28. Yan SY, Hu Y, Fan JG, Tao GQ, Lu YM, Cai X, et al. Clinicopathologic significance of HER-2/neu protein expression and gene amplification in gastric carcinoma. World J Gastroenterol. 2011;17:1501-6.

29. Im SA, Kim JW, Kim JS, Kim MA, Jordan B, Pickl M, et al. Clinicopathologic characteristics of patients with stage III/IV $(\mathrm{M}(0))$ advanced gastric cancer, according to HER2 status assessed by immunohistochemistry and fluorescence in situ hybridization. Diagn Mol Pathol. 2011;20:94-100.

30. Boers JE, Meeuwissen H, Methorst N. HER2 status in gastrooesophageal adenocarcinomas assessed by two rabbit monoclonal antibodies (SP3 and 4B5) and two in situ hybridization methods (FISH and SISH). Histopathology. 2011;58:383-94.
31. Moelans CB, Milne AN, Morsink FH, Offerhaus GJ, van Diest PJ. Low frequency of HER2 amplification and overexpression in early onset gastric cancer. Cell Oncol (Dordr). 2011;34:89-95.

32. Lee OJ, Kim HJ, Kim JR, Watanabe H. The prognostic significance of the mucin phenotype of gastric adenocarcinoma and its relationship with histologic classifications. Oncol Rep. 2009;21: 387-93.

33. Levine AJ. p53, the cellular gatekeeper for growth and division. Cell. 1997;88:323-31.

34. Oren M. Lonely no more: p53 finds its kin in a tumor suppressor haven. Cell. 1997;90:829-32.

35. Yano T, Doi T, Ohtsu A, Boku N, Hashizume K, Nakanishi M, et al. Comparison of HER2 gene amplification assessed by fluorescence in situ hybridization and HER2 protein expression assessed by immunohistochemistry in gastric cancer. Oncol Rep. 2006;15:65-71.

36. Albarello L, Pecciarini L, Doglioni C. HER2 testing in gastric cancer. Adv Anat Pathol. 2011;18:53-9. 